FERMILAB-Pub-83/31 2000.000

MAGNETIC MONOPOLES: A STATUS REPORT

R. A. Carrigan, Jr.

Fermi National Accelerator Laboratory, Batavia, Illinois

and

W. P. Trower

Virginia Polytechnic Institute and State University, Blacksburg, Virginia

March 1983 


\title{
MAGNETIC MONOPOLES: A STATUS REPORT
}

\author{
Richard A. Carrigan, Jr. \\ Fermi National Accelerator Laboratory \\ Batavia, Illinois 60510 \\ W. Peter Trower* \\ Physics Department \\ Virginia Polytechnic Institute and State University \\ Biacksburg, Virginia 24061
}

\section{ABSTRACT}

A solitary, uncorroborated Stanford candidate event is the only evidence that magnetic monopoles might exist. Powerfur theoretical motivation for monopoles derives from Dirac's assertion that monopoles could explain charge quantization and the ' $t$, Hooft-Polyakov demonstration that monopoles are an inevitable consequence of many gauge theories currently being used to unify the electroweak (photon-lepton) and nuclear (quark) interactions. The monopole abundance implied by the Stanford event is in clear contradiction to bounds on their number from astronomical data. Fortunately, the already considerable and expanding arsenal of detection techniques are being fashioned to experimentally test the many open questions surrounding monopoles.

*Work supported in part by grants from the National Science Foundation
(PHY-80-20411) and the Jeffress Trust. 


\section{INTRODUCTION}

The flush of excitement caused by Cabrera's candidate magnetic monopole event reported last spring ${ }^{1}$ has been reduced to a hush of activity as experimenters around the world contrive and construct detectors. One of their objectives is to corroborate or refute this solitary bit of evidence that is tantalizingly linked with the validity of the current principal theory of matter. This activity has become the focus of many scientists from formerly weakly connected disciplines: low temperature and high energy experimentalists; particle theorists, cosmologists and astrophysicists. Such a mixture has produced an excitement characteristic of nascent science before it is subdued into well-behaved formality.

This status report reviews the developments in the field of magnetic monopoles during the past year. ${ }^{2}$. The situation which preceded the Stanford Valentine's Day event is summarized in order to place this important development in context. Next, the recent and traditional experimental techniques and the results which they have produced are described. The important question of where monopoles, if they exist, should and shouldn't be found is reviewed. Finally, the implications for theory of the experimental results, both actual and anticipated, are summarized; and opportunities for experiments provided by the development of new theoretical ideas are outlined. ${ }^{3}$ 


\section{THE HISTORY}

Perhaps the earliest recorded discussion of magnetic monopoles ${ }^{4}$ is contained in a letter written by Petrus Peregrinus de Maricourt, a French military engineer, in $1269 \mathrm{AD}$. The first glimmerings of the idea of poles and lines of force are contained there. The great magnetist, Gilbert, was acquainted with the Peregrinus investigations. Later Maxwell considered magnetic poles in his famous unification of electrical and magnetic phenomena, but they did not survive to his final formulation as there was no experimental justification for their inclusion. ${ }^{5}$

Despite brief flirtations by such giants as P. Curie, ${ }^{6} \mathrm{~J} . \mathrm{J}$. Thomson, ${ }^{7}$ and $\mathrm{H}$. Poincare, ${ }^{8}$ monopoles were largely ignored by the scientific community until 1931. In that year P.A.M. Dirac ${ }^{\circ}$, fresh from the triumphant marriage of quantum mechanics and special relativity, turned to a quantum-mechanical study of a problem first addressed classically by J.J. Thomson: that of the motion of an electric charge in the field of a magnetic monopole. Thompson had noticed the remarkable fact that the electromagnetic angular momentum in a magnetic pole-electric charge system was independent of their separation. Dirac's result is the now famous quantization condition:

$$
\text { eg }=n(\hbar c / 2) \text {, }
$$

where $e$ and $g$ are, respectively, the electric and magnetic charges and $n$ is the principal quantum number. In effect, the monopole charge is 70 times the electric charge, just half the inverse fine structure constant $l_{\alpha}=\mathrm{e}^{2} / \mathrm{hc}=$ 1/137) which characterizes the strength of electromagnetic interactions. 
Stated most dramatically, Dirac's quantization demonstrates that a single magnetic pole existing anywhere in the universe would explain the fact that all electric charge occurs only as discrete integral multiples of e, regardless of the other properties of a host particle. Thus, monopoles explain the mystery of charge quantization. Dirac theory is moot on other monopole properties: size, shape, mass, parity, spin, statistics, sources and abundance.

Two consequences of magnetic charge, with its field seventy times stronger than that of an electric charge, were immediately recognized. First, a rapidly moving monopole should produce heavy ionization as it passes through matter. Theory suggests that a relativistic monopole should be 5000 times as heavily ionizing as an electron. Second, monopoles would bind to some forms of matter such as ferromagnetic domains.

Implicit in all the hopeful but unproductive experiments during the ensuing decades was the assumption that the monopole mass was not too much 1 arger than those of other particles.10 Such monopoles could attain velocities approaching that of light and could be seen in optical detectors by the bremsstrahlung, de-excitation, Cherenkov, and/or transition photons which they produce. Because of the expected high energy loss, these monopoles would be brought to rest in matter more readily than their electrically charged counterparts. Once sedentary, monopoles would append themselves to matter from which they could be dragged with sufficently strong pulsed magnetic fields. Finally, although these monopoles could be found in cosmic rays, they might also be produced by an accelerator with suitably high energy. 
In 1975 one event from a high altitude plastic-emulsion detector exposure taken during a balloon flight was claimed to have been produced by the passage of a monopole.11 Its interpretation was quickly challenged on the grounds of experimental problems, incompatibilites with other experiments and possibilities for a less exotic cause. 12

The idea of looking directly for manifestations of the magnetic charge using electromagnetic induction was conceived and tested in the sixties. ${ }^{3}$ The passage of a monopole through a closed conducting ring should induce a current change intent on maintaining the ring's pre-monopole magnetic flux. Some of the original experiments were done with room temperature conducting coils. With the advent of superconducting rings in which a current change would persist indefinitely, undegraded by Joule heating characteristic of more mundane materials, these experiments became easier to carry out. The low sensitivity of current measuring electronics in the early superconducting devices required many passages of a monopole bearing sample to produce a palpable signal, so this technique was initially restricted to bulk matter searches, including one where many kilograms of lunar material was found to be magnetically neutral to less than a twentieth of a Dirac charge. ${ }^{4}$ However, with the development of the Superconducting QUantum Interferometer Device, SQUID, and U1tra-low magnetic field shields, a single pass and, therefore, a dynamic monopole detector became possible. ${ }^{15}$ 
Earlier in 1974 a profound theoretical insight was uncovered that would have revolutionary consequences for contemporary monopole searches. Polyakov and ' $t$ Hooft ${ }^{16}$ independently showed that monopoles appear as stable solutions of the spontaneously broken Yang-Mills field equations and were required by a large class of theories. ${ }^{17}$ Goldhaber ${ }^{18}$ calls the links between these broken gauge theories, charge quantization and magnetic monopoles "the Golden Triangle." These non-Abelian gauge theories had broad general application to the description of elementary particles. Ironically, the gauge theory evoked in the successful unification of the weak, SU(2), and electromagnetic, $U(1)$, interactions was not one of these. Nor was the gauge theory responsible for the also successful Quantum Chromodynamics, QCD, which described the nuclear force. However, non-Abelian gauge theories found employment in the synthesis of electroweak and strong interactions, the so-called Grand Unification Theories, GUTs.

In a typical GUT model there is no difference between strong, weak and electromagnetic forces at a high enough temperature because there is perfect symmetry. This temperature, or energy, is typically $10^{15} \mathrm{GeV}$. As the temperature is lowered, it is possible to have a non-zero value for the vacuum expectation of the scalar Higg's field. Breaking the symetry freezes in a fixed space direction. In cosmology this direction can only span a distance equal to the velocity of light times the age of the universe. In the early universe these domains were very small. A GUT monopole can be viewed as the coalescence of several of these domains to form the magnetic field distribution of a pole as shown in Fig. $1 .{ }^{19}$ The monopole is heavy because the domains are confined to a space that is $10^{-28} \mathrm{~cm}$ on a side. 
All of GUT particle physics can be rolled up in a 't Hooft monopole. Near the center, $10^{-29} \mathrm{~cm}$, there is a GUTS symetric vacuum populated with virtual grand unification particles. Much further out, $10^{-16} \mathrm{~cm}$, the field is color-electroweak with $Z$ and $W$ bosons. At a Fermi, $10^{-13} \mathrm{~cm}$, it is color magnetic and electromagnetic with gluons and photons. Beyond nuclear distances it behaves solely as a magnetic monopole as seen in Fig. 2.

The current view of the GUT monopole raises the possibility that when it passes through matter, a collision with a proton may occur in which the monopole's core is overlapped, causing the proton to spontaneously change into a pion and a positron. In other words, proton decay, which must ordinarily be exceedingly rare, may be catalyzed in the presence of a monopole. The possibility of observing this effect, called baryon catalysis or the Rubakov-Callan effect, ${ }^{20}$ has attracted much interest. Theoretical opinions on the possibility of catalysis are widely divergent. ${ }^{21}$

Polyakov-'t Hooft monopoles possess the disconcerting property that they be enormously massive compared to run-of-the-mill particles, around $10^{16}$ $\mathrm{GeV} / \mathrm{c}^{2}$. A proton's mass is approximately one $\mathrm{GeV} / \mathrm{c}^{2}$. Such massive monopoles could only have been produced in the first instants after the creation of the universe in the Big Bang. Standard cosmology and reasonable GUT theory suggest that the number of monopoles roughly equal the number of nucleons. ${ }^{22}$ On the other hand, it appears that nucleons account for much of the matter in the universe. The presence of a galactic magnetic field implies even fewer monopoles. ${ }^{23}$ Thus by $1981,{ }^{24}$ it was argued that the flux of monopoles would be no greater than $10^{-16} / \mathrm{cm}^{2} / \mathrm{s} / \mathrm{sr}$. 
Because GUT monopoles are expected to be very massive, they would necessarily be slow if they were to be contained in the galaxy, would rarely be stopped and if somehow trapped in matter, would easily be dislodged by even modest accelerations. For example, such a monopole moving at $1 / 100$ of the speed of light could easily penetrate through the earth. The most powerful electromagnet, one kilometer long, would change the monopole direction less than a hundred millionth of a degree. Thus, this one idea explained why none of the experiments since Dirac's prediction could have seen a magnetic monopole. Further, it left only one detection technique free of questions -that employed by the dynamic induction detector.

The candidate Stanford event of February 1982 was recorded in such an induction detector. The event possessed characteristics consistent with the passage of a particle with magnetic charge of $g$ to $\pm 5 \% .^{1}$ The instrument which detected this monopole candidate, shown in Fig. 3 , was a magnetically shielded magnetometer. The superconducting detector loop was shielded with a sophisticated superconducting shield that held ambient magnetic fields to less that $10^{-7} \mathrm{G}$, among the lowest ever obtained. Baseline noise in the Cabrera system was typically $1 \%$ of a monopole offset signal. Although this signal is not easily attributable to any other probable cause, its possible origin in the release of some internal instrumental stress cannot be entirely ruled out. Without a second event being seen to date, this experiment has now set an upper limit of $6 \times 10^{-11}$ monopoles $/ \mathrm{cm}^{2} / \mathrm{s} / \mathrm{sr}$ for the isotropic distribution of particles with charge greater than $0.06 \mathrm{~g}$. This is a hundred thousand larger than the earlier expected upper limit. ${ }^{23}$ 
An induction detector has been used to examine the superconducting niobium spheres $^{15}$ on which Fairbank and collaborators ${ }^{25}$ have reported finding fractional electric charges. Measurements made over a decade on the niobium spheres have failed to reveal any magnetic charge. These searches were motivated by Schwinger's ${ }^{26}$ proposal in the mid-sixties that combined both electric and magnetic charge into an object called a dyon. Experimental searches for dyons have been equivalent to those for magnetic monopoles. ${ }^{27}$ 


\section{DETECTABLE PROPERTIES}

The presence and properties of a monopole can be deduced from their unique interactions with matter. Some techniques that have long been used for successful detection of electrically charged particles are of questionable utility for GUT monopoles. The discussion will center first on an approach which can uniquely detect superheavy monopoles.

Induction.

When the magnetic flux through a conducting ring is changed, a current is induced in the ring. This ring current is quickly extinguished by the resistance of the ring material except in the case where a superconductor is used. For a superconducting ring the macroscopic quantum mechanical effect must be included using the Ginzberg-Landau formulation. ${ }^{28}$ The supercurrent density is

$$
\vec{j}_{e}=\frac{h e^{\star}}{2 i m^{\star}}\left(\psi^{\star} \vec{\nabla} \psi-\psi^{\vec{\nabla}^{\star}} \psi\right)-\frac{e^{\star_{2}}}{m^{\star} c} \psi^{\star} \psi \vec{A},
$$

where the particles involved are Cooper pairs whose mass and electric charge are twice that of the electron and $A$ is the electromagnetic vector potential. $\psi$, the coherent many-body state of cooper pairs, has a local pair density, $\psi^{*} \psi=n_{s} / 2$, which is half the superelectron density, $n_{s}$. This equation can be solved ${ }^{15}$ in conjunction with Maxwell's equations for a closed loop to show that the magnetic flux through the ring must be an integer number of 


$$
\phi_{0}=(\mathrm{hc} / 2 \mathrm{e})=207 \mathrm{nG} \cdot \mathrm{cm}^{2} \text {, }
$$

the superconductivity flux quantum. For a magnetic monopole passing through the loop

$$
\phi(t)=\phi_{S}(t)+\phi_{g}(t)=-4 \pi g \theta(t),
$$

where $\phi=0$ at $t=-\infty$ is assumed. $\theta$ is the half angle subtended by the ring at the monopole. The first term, the self-induced ring flux, is related to the supercurrent by

$$
\phi_{S}(t)=-I(t) L,
$$

where $L$ is the ring self-inductance. The second term is the monopole flux coupling the ring

$$
\phi_{g}(t)=2 \pi g\left(1-2 \theta(t)+\gamma t\left((\gamma v t)^{2}+a^{2}\right)^{-\frac{1}{2}}\right),
$$

where $\gamma=\left(1-v^{2} / c^{2}\right)^{-\frac{1}{2}}, v$ is the velocity of the monopole and $a$ is the loop radius. The induced ring current is

$$
I(t)=\left(\phi_{0} / L\right)\left(1+\gamma v t\left((\gamma v t)^{2}+a^{2}\right)^{-\frac{1}{2}}\right) .
$$


Figure 4 illustrates the ring current change for several monopole trajectories. Cabrera, ${ }^{27}$ in Fig. 5, has given a nice illustration of the actual process of the monopole pulling flux through the loop to induce a toroidal field around $i t$. The characteristic time, $a / \gamma v$, is $s 100 \mathrm{~ns}$ for $\beta$ $\sim 10^{-3}$ for $a s$ few centimeters. For particles passing through the ring, a persistent change in ring current will only occur for a penetrating magnetic charge, while a penetrating electric charge or magnetic dipole, as well as a non-penetrating magnetic charge, will cause only small, transient current changes. Notice also that the sign of the current depends on the polarity of the magnetic pole and its direction of passage through the loop.

of course, a change in any ambient magnetic flux through the loop will also induce a current change. Most recent inductive experiments have used superconducting shields to ameliorate this possibility. In essence, such a shield freezes the ambient field in place. It also interacts with the loop and modifies the response, depending on the distance of the shield from the loop. By the same token, the shield should cause somewhat smaller offset current events in the loop for monopole trajectories that don't go through the 10op. Some detection schemes anticipate using this effect to increase the effective detection area.

It is possible the Cabrera candidate event was caused by an ambient flux change through the loop although Cabrera's superconducting shield is the best in the world. Other new searches using this approach have opted for less sophisticated shielding in order to accommodate larger loops. Gradiometers ${ }^{29}$ or macromes ${ }^{30}$ are used to minimize this effect. Most of these detectors, such as Cabrera's triaxial detector, incorporate multiple loops for 
redundancy, possible direction measurement and increased detection area. Non-superconducting techniques have also been discussed. ${ }^{31}$ The combination of a superconducting loop, shield and SQUID appears in practice to provide the best sensitivity.

Cabrera has also suggested scanning the shield with a sensitive magnetometer to detect the twin magnetic vortices left by the passage of a magnetic monopole.

Ionization.

Several processes involving electromagnetic interactions of electrically charged particles result in detectable photons ${ }^{32}$ and a concomitant energy loss for the particle. These include bremsstrahiung, Cherenkov light, transition radiation, and ionization or excitation of an atom.

The analog interactions for magnetic monopoles benefit from the stronger magnetic charge but suffer from the expectation that a massive GUT monopole will have a velocity much smaller than that of light.

In general, energy loss is a function of $\beta$, the ratio of the particle velocity to the velocity of light. Detailed calculations of the photon-producing processes for magnetic monopoles conclude that atomic de-excitation alone holds any promise for monopole detection. The estimates for energy $10 \mathrm{~s}^{33}$ at $\beta$ of $10^{-3}$ expected for a GUT monopole differ by four orders of magnitude. This uncertainity not only bears on the possibility of detecting monopoles in various media but also relates to the possibility of 
their being trapped in astronomical bodies since their range will depend on the rate at which they lose energy. On the other hand, ionization offers a much better possibility than induction for devising extremely large detectors.

The origin of this uncertainity in energy loss estimates lies in the fact that atomic collisions are complicated. For relativistic charged particles, these inherent complications are simplified by arbitrarily separating the interaction into two soluble classes: close collisions and distant collisions. In close collisions the energy transfers are so much larger than the electron's binding energy that the electron is considered free and the impulse approximation is satisfactory. The energy loss is then easily calculated from simple kinematics and the scattering cross sections. In distant collisions, the atom is considered to be excited by the perturbing electric field of the glancing particle and the dipole approximation is used.

These calculations break down when the velocity is sufficiently small so that most of the collisions no longer fall into only one class. Then a real model for the atom must be used to understand the dynamics. The actual calculation requires approximations be made in order to produce a result. Some years ago, Lindard ${ }^{34}$ worked out this theory for protons. Experiments done with protons down to $\beta^{\prime} s$ of approximately $10^{-3}$ are in good agreement with theory. Ahlen and Kinoshita have applied this theory to monopoles to produce some of the most credible energy loss estimates. The results are shown in Fig. 6 . 
Recently, energy loss to Zeeman splitting at low velocities in hydrogen has been studied from a fundamental basis. ${ }^{35}$ Estimates of energy loss at low velocity to this mechanism in helium and hydrogen are substantially higher than ionization losses in silicon.

An interesting possibility is a combination of an induction and ionization detector. If a monopole passes through such a detector and no ionization is detected within a few milliseconds of the induction event, the credibility of the ionization loss estimates and/or induction signal is called into question. Perhaps the monopole velocity is much slower than previously thought, implying an even heavier monopole. If attendant ionization is discovered, the monopole speed and direction could be determined to give the polarity of the magnetic charge.

Ionization experiments which use either plastic scintillation counters coupled to photomultiplier tubes of proportional ionization chambers have several detector planes spaced meters apart to signal the coordinates and time of passage of a monopole candidate event. For GUT monopoles these signals would be separated in time by several microseconds. Cosmic ray events are the overwhelming source of background, so placing the detector deep underground will reduce this background. At lower velocities the energy loss is expected to be smaller, so there is a premium on making the detectors sensitive to small ionization losses. 
Acoustical Signals.

A dozen years ago Hofstader ${ }^{36}$ suggested and demonstrated experimentaliy that electron beams could produce mechanical oscillations. Such signals have also been produced by protons. ${ }^{37}$ Despite considerable interest in the possibilities, no particle detector has been developed using this principle due to the poor signal-to-noise ratio. So far, only bunches containing many particles, each with an energy deposition of approximately $2 \mathrm{MeV} / \mathrm{cm}$, have produced a detectable thermoacoustic wave. Encouraged by early estimates of monopole energy loss of $2 \mathrm{GeV} / \mathrm{cm}^{3{ }^{3}}$ interest in this possibility for monopole detection has been renewed. However, calculations of the thermal fluctuation pressure suggest that this severely limits the thermoacoustic detection of monopoles for conductive media with temperatures above a few millidegrees. ${ }^{39}$ Noise sources attendant to actual acoustic measurements are also discouragingly large. Experimental work exploring acoustical detection is being pursued. 40

Electromagnetic Evidence.

During the nineteen fifties, five emulsion events with common properties seen by three different groups in balloon flights defied explanation in terms of known physics. ${ }^{41}$ Their unusual property was that only electron-positron pairs were present. These pairs appeared in great number and in a configuration which indicated a very energetic process, certainly above a few TeV. Finally, each event began deep in the emulsion with no track of a causal particle in evidence. 
A decade and a half later it was proposed that these unusual events could have been the results of the photoproduction of a virtual monopole-antimonopole pair. ${ }^{42}$ The photon shower which pair-produces electrons was the consequence of bremsstrahlung and annihilation radiation. Accelerator searches have failed to discover any of these pure multiphoton showers. ${ }^{43}$

With the advent of the GUT monopole the monopole-antimonopole system, now familiarized to monopolonium, has been revisited ${ }^{44}$ with some amazing consequences. The lifetime of monopolonium ranges from days to as long as hundreds of billions of years. At a separation of an Angstrom the velocity of the monopole in orbit is $1 \mathrm{~cm} / \mathrm{sec}$, the principal quantum number is roughly a trillion, but the binding is still $40 \mathrm{keV}$. Monopolonium decays by classical Larmor radiation for all but the last 10 seconds of its life. It de-excites first by emitting radio, then successively light, $x$-rays, $\gamma$-rays, quarks and gluons, intermediate bosons, and ultimately $10^{14} \mathrm{GeV} / \mathrm{c}^{2} \mathrm{X}$ particles. Typically some tens of millions of particles are emitted altogether. Monopolonium radiation at a wavelength of $1 \mathrm{~cm}$ is expected to give a flux of $10^{-24} / \mathrm{eV} / \mathrm{cm}^{2} / \mathrm{s} / \mathrm{Hz}$. Current observational limits are $3 \mathrm{x}$ $10^{-16} / \mathrm{eV} / \mathrm{cm}^{2} / \mathrm{s} / \mathrm{Hz}$, corresponding to 50 microjanskies. In general, the prospects for other monopolonium products are equally dim. 
SOURCES AND SINKS OF MONOPOLES

Possible sources and sinks of GUT monopoles (with relevant problems) inciude: the Big Bang (inaccessible), monopolonium (rare), the galaxy (extant magnetic fields), between clusters of galaxies (hard to reach), the surface of the earth (improbable), the sun (hard to tell), the interior of the earth (1ess likely than the sun), meteorites (braking problems), accelerators (too puny), cosmic rays (rare), made by cosmic rays (still too puny) and in detectors searching for proton decay terrestrially and in neutron stars (no theoretical agreement on monopole catalysis). These monopole sources and sinks are considered, moving from the nearby space-time to the Big Bang.

\section{Accelerator Searches.}

Traditionally, particle searches are most successfully carried out at accelerators. Because of the unusual properties of monopoles, it is possible to devise accelerator experiments that are background free. The experiments proceed by bombarding a target with a proton or electron beam and either trapping or directly detecting any monopoles that are produced. Colliding beams of particles and antiparticles have also been examined for evidence of monopoles. 
Figure 7 shows the modern upper limits on the cross section for accelerator monopole searches including measurements made recently at the CERN SPS collider ${ }^{45}$ and at PEP. ${ }^{46}$ No monopoles have been found in the accelerator searches. Indeed, in all cases, these cross section limits are typically the smallest measured at an accelerator. However, it is now clear that such searches can shed no light on the GUT monopole. The absolute maximum mass monopole that can be produced in a particle-antiparticle collision is the energy of one of the incident particles, $270 \mathrm{GeV}$ in the case of the CERN SPSC. Gauge theories with monopoles suggest a lower limit on the monopole mass, the Bogomol 'nyi bound, ${ }^{47}$ which is greater than the mass of the gauge boson associated with the theory divided by the appropriate coupling constant. For standard electroweak theory, with an intermediate boson of 70 or $80 \mathrm{GeV} / \mathrm{c}^{2}$ and the fine structure constant, the monopole mass would be $10,000 \mathrm{GeV} / \mathrm{c}^{2}$. No known accelerator technology can reach energies of $10^{16}$ GeV needed to produce grand unification monopoles. Thus, accelerator monopole searches are no longer in the mainstream, but they will continue to be done since other unexpected production mechanisms might turn up for monopoles that don't derive from grand unification.

Cosmic Ray Searches.

The cosmic ray flux at the surface of the earth is $0.01 / \mathrm{cm}^{2} / \mathrm{s} / \mathrm{sr}$. The present Cabrera flux limit is equivalent to a hundred-millionth of this. 
Theoretical estimates of monopole fluxes ${ }^{24}$ suggest values of from $10^{-15}$ to $10^{-17} / \mathrm{cm}^{2} / \mathrm{s} / \mathrm{sr}$. Such fluxes require football field size detectors operating for years. The largest area-time factor reported for an ionization search is from the Baksan neutrino detector, ${ }^{48}$ which has produced a limit on the flux nearly ten thousand times lower than the current Cabrera limit. A summary of these 1 imits ${ }^{49}$ is given in Fig. 8.

Cosmic rays can also produce elementary particles through interactions. Al though cosmic ray energies range up to $10^{11} \mathrm{GeV}$, this is still too small to produce GUT monopoles. Thus, monopoles found in cosmic rays will be primordial. This is one of several reasons for skepticism. Monopolonium may also produce multiple extended air shower events in the large Fly's Eye detector in Utah.

Matter Searches.

Monopoles may be present in matter either because they were accreted there when the matter formed or because they stopped after losing their kinetic energy. Stopped monopoles can bind to ferromagnetic or paramagnetic material through image charges. ${ }^{56}$ Some argue monopoles may bind to atomic nuclei. ${ }^{57}$ One obvious possibility is to search iron from the surface of the earth. However, the expected kinetic energy of a cosmic GUT monopole is such that it could stop anywhere in the earth's interior, if it stopped at a11. As a result, the number of monopoles per unit volume should be small and uniform through the earth. One suggestion is to look for monopoles falling under gravity out of iron refinery operations, ${ }^{57}$ where millions of tons of surface ore is raised above the curie point every year in the 
manufacture of steel.

Monopoles accreted in planetary formation would probably reside nearer the core of the earth since the force of gravity would overcome any sustaining force of matter. For GUT monopoles, gravitational forces are typically more significant than magnetic forces. Limits on the monopole population have been set by considering the possibility of monopole-antimonopole annihilation deep in the earth contributing to the known heat flux from the earth. 58 Detailed consideration of the de-excitation mechanism shows that this is probably' a very slow process. The earth's heat flux has al so been used to set limits on the monopole catalysis mechanism. 59

Sources in the Solar system.

Meteors, with their smaller gravity, might provide safe harbor for monopoles. ${ }^{0}$ Unfortunately, a meteor-trapped monopole that hits the earth will continue umimpeded because it carries far more momentum than the normal atoms and would shear right through matter. A possible alternative is to carefully capture a meteor in space and search there.

The sun has also been suggested as a source for monopoles. ${ }^{61}$ This scenario evolved principally to explain the unexpectediy high flux suggested by the Cabrera event. In this picture the sun contains $10^{26}$ monopoles and emits $10^{9}$ monopoles/second over its lifetime. The sun must possess an interior magnetic field of about $1 \mathrm{kG}$ to prevent monopoles from falling to the center as they undergo ohmic energy losses. The solar flare fields expel monopoles with velocities similar to the earth's velocity around the sun, $30 \mathrm{~km} / \mathrm{s}$, so 
that they form a cloud in the earth's orbit. on the average, these monopoles would have velocities five times smaller than monopole galactic velocities at the surface of the earth. Large temporal flux variations as seen in the solar wind could be present. This model predicts an 11 year sun spot cycle, the only suggested mechanism which explains this phenomenon. The sun, because of its gravitational potential, could act as a monopole concentrator if a mechanism by which they lose energy could be established. The concentrating effect for GUT monopoles in the sun has been calculated to be well below that needed to achieve the posited solar population, 62 suggesting that a solar monopole level of this magnitude would have to be primordial. This is one of several reasons for skepticism.

A few observations of the sun suggests that it has a magnetic monopole moment. ${ }^{63}$ Taken at face value, ${ }^{64}$ these measurements are consistent with a net North monopole abundance of about $10^{29}$. Many other more prosaic explanations have been offered for these observations. 65

The presence of solar monopoles could provide an additional mechanism for solar heat transport. ${ }^{66}$ Monopoles could also catalyze fusion of magnetically dipolar nuclei, ${ }^{64}$ the ${ }^{3} \mathrm{He}+{ }^{3} \mathrm{He}$ reaction (but not the ${ }^{3} \mathrm{He}+{ }^{4} \mathrm{He}$ reaction), thus explaining the low solar flux of certain neutrinos. ${ }^{6}$ If monopole catalysis was present, there would also be a change in the solar heat balance and possibly a detectable flux of hundred MeV neutrinos at the earth. ${ }^{61}$ 
Monopole baryon catalysis offers an interesting possibility for terrestrial as well as astronomical observations. In SU(5) grand unification, the proton lifetime is expected to be about $10^{31}$ years so that its detection requires large amounts of matter. On the other hand, a monopole passing through a proton decay detector could catalyze with a strong interaction cross section, inducing some tens of decays. Since monopoles are expected to be slow, all the detector electronic gates must be kept open during the monopole passage, typically for fifty microseconds. The upper limit on the monopole flux is currently less than $5 \times 10^{-15} / \mathrm{cm}^{2} / \mathrm{s} / \mathrm{sr}$ in the absence of any observed events and assuming the cross section to be about $100 \mathrm{mb} .^{68}$

\section{Astrophysical Sources.}

Neutron stars should be relatively good monopole collectors. ${ }^{69}$ once inside a star, catalyzing monopoles could transform nucleons at such a rate that the resulting $x$-ray luminosity would exceed by many orders of magnitude the measured upper limits of neutron star x-ray luminosities. This implies a monopole flux, assuming hadronic cross sections for catalysis, less than $5 x$ $10^{-22} / \mathrm{cm}^{2} / \mathrm{s} / \mathrm{sr}$, much lower than any other flux limit. Alternatively, one can put in flux upper limits and deduce an upper limit for the catalysis cross sections. There are still questions about the heat transfer mechanism inside a neutron star, the catalysis rate, the microenvironment surrounding a monopole, possible recombination of monopole pairs and the superconducting interior of a neutron star which bear on this argument. ${ }^{70}$ A similar analysis has been made for a peculiar subclass of A stars. ${ }^{11}$ 
In the galaxy monopoles are occasionally called upon to supply the "dark matter" which appears to be necessary to describe its dynamics. Monopoles could supply such mass without producing much radiation. A monopole halo could also prevent the so-called bar mode plasma instability that occurs for a flat galactic structure. ${ }^{72}$

Central to the question of galactic magnetic monopole abundance is the so-called "Parker bound." ${ }^{3}$ Free monopoles in a magnetic field will neutralize the field since the electric currents that generate the field have to do work on the monopoles, thereby dissipating the currents. The lifetime in seconds of a cosmical magnetic field in the presence of monopoles is:

$$
\tau=\frac{B}{8 N_{g} g V}=\frac{B}{8 g F}
$$

where $B$ is the magnetic field, typically $3 \mu G ; N_{g}$ is the free monopole density in $\mathrm{cm}^{-3}$; and $F$ is the monopole flux. The regeneration time of the galactic field is approximately 30 million years. This implies that the flux limit for free monopoles is less that $10^{-16} / \mathrm{cm}^{2} / \mathrm{s} / \mathrm{sr}$, or roughly one hundred thousand times smaller than the Cabrera flux. This is, of course, the flux for free monopoles not bound in some way or near a monopole source.

Recently this limit has been re-examined in detail as a function of monopole mass and velocity. ${ }^{73}$ Even for monopoles with masses of $10^{19} \mathrm{GeV} / \mathrm{c}^{2}$, near the Planck mass, it is difficult to achieve fluxes higher than $10^{-12} / \mathrm{cm}^{2} / \mathrm{s} / \mathrm{sr}$. 
One escape from the Parker bound could be that the galactic magnetic field is due to monopoles. ${ }^{72,74}$ In this picture, clouds of north and south monopoles oscillate through each other to produce an alternating field. Rather than depleting the magnetic field, the monopoles transfer energy back and forth from kinetic energy to magnetic field energy. However, if the galactic field were produced by magnetic monopoles, it should have a vanishing curl which contradicts observation. Indeed, the magnetic field configuration and magnitude generally fits the picture of a dynamo. The relatively short field oscillation time for a monopole galactic field would prevent dust grain alignment, ${ }^{75}$ a process known to exist in the galaxy from star light polarization measurements. The short oscillation time also leads to difficulties in confining cosmic rays. Some of these problems can be ameliorated by different choices of monopole masses, but these challenges to the Parker bound do not seem to fit the general picture of the galactic magnetic field distribution. ${ }^{76}$

Parker bound arguments can be extended to the impact of monopoles on extragalactic magnetic fields. There is evidence for intragalactic cluster fields of the order of $10^{-7}-10^{-8} \mathrm{G}$. Other observations, such as a possible enhancement of high-energy cosmic rays from the Virgo cluster, indicate that extragalactic fields cannot be much larger than this. These low fields can be used to infer flux limits in our galaxy a thousand times lower than the galactic Parker bound. ${ }^{77}$ 
The Big Bang.

The conundrum of monopoles in the Big Bang has already been mentioned. ${ }^{22}$ Standard cosmology and SU(5) grand unified theories suggest a monopole number roughly the same as the proton number in the universe. The estimated total protonic mass in the universe is not far from that needed to close the universe. If an equivalent number of monopoles with their enormously large mass is added, the universe would have closed far too quickly. This implies that there can't be as many monopoles as naive cosmological estimates would indicate. However, more complicated models such as so(10) can overcome this problem and predict about one monopole for every $10^{20}$ protons. ${ }^{78}$

of the ways to reduce the number of monopoles, perhaps the most intriguing is "the inflationary universe" hypothesis. ${ }^{79}$ In essence, the universe expands exponentially and supercools. The actual expansion is enormous. This process erases the previous history of the universe. After the phase transition occurs, the universe is supposed to reheat and evolve along the lines of the standard Big Bang. With one stroke this model severely depresses monopole production and exprains the flatness and the horizon problems.

There is a serious problem -- there is no way to escape from the inflationary phase. However, a modified GUT approach using the Coleman-Weinberg ${ }^{80}$ potential might allow the inflationary phase to gradually evolve into the current universe. ${ }^{31}$ Several problems remain, the most serious being the apparent absence of any realistic GUT model in which the scenario can be implanted. For example, some claim that this picture is so smooth that there 
is no galaxy formation. One possible way out is provided by the supersymmetric model, called SUSY-GUTS, which relates fermions and bosons. ${ }^{82}$ Unfortunately, there are no known super-symmetric partners of established particles. 


\section{THE FUTURE}

As a result of much recent activity, the theoretical picture of the GUT monopole has become clearer. Newer ideas like monopolonium and monopole catalysis of baryon decay remain unsettled.

The Cabrera event has stimulated a number of inductive searches. However, none of these, including Cabrera's improved detector, have seen a second event so that the flux limit is now an order of magnitude lower than in 1982. If in the next year no other event is seen, the flux limit will probably be reduced by an additional factor of a hundred by these experiments, still a thousand times less sensitive than the nominal Parker bound. These experiments appear to be the most reliable way of unambiguously detecting a monopole and the only way of sensing its polarity.

The recent theoretical work on ionization of monopoles in matter has put ionization experiments on a firmer footing down to $\beta^{\prime} s$ of $10^{-3}$. Nevertheless, there are significant contradictions and uncertainties that must be taken into account when planning and evaluating ionization experiments. These problems are balanced by larger detection areas. Many large scale ionization searches are now under way. If these produce null results, then the largest can come close to the nominal Parker bound. 
The best opportunity to answer monopole questions may lie within the grand scale of cosmology and astrophysics. Limits such as the Parker bound and neutron star catalysis need to be pressed observationally. Even more interesting would be evidence of some large scale positive artifact.

Much more theoretical work and, hopefully, some observational tests are needed to clarify the creation and survivability of monopoles in the universe.

\section{ACKNOWLEDGEMENTS}

Much of the work reviewed here was presented at the Wingspread Monopole Workshop held in Racine, Wisconsin. That Workshop was supported by the Johnson Foundation, the NATO Advanced Research Workshops Programme, the U.S. Department of Energy, and the U.S. National Science Foundation. The authors wish to thank the participants at the Wingspread Workshop for a great deal of interesting information. R.E. Craven has collaborated in both the organization of the Workshop and the preparation of this review. Support for this work was provided WPT in part by grants from the Jeffress Trust and the NSF (PHY-80 20411). 


\section{REFERENCES}

1. B. Cabrera, Phys. Rev. Lett. 48, 1378 (1982).

2. For an earlier review see B. Cabrera and W.P. Trower, Found. Phys. 13, 195 (1983).

3. A more complete discussion of the material contained here will appear as Magnetic Monopoles, edited by R.A. Carrigan, Jr. and W.P. Trower (Plenum, New York, 1983). To be published.

4. The Letter of Petrus Peregrinus on the Magnet, A.D. 1269, translated by Brother Arnotd (McGraw, New York, 1904).

5. C.W.F. Everitt, private communication.

6. P. Curie, Seances Soc. Phys. (Paris) (1894) p. 76.

7. J.J. Thomson, Elements of the Mathematical Theory of Electricity and Magnetism (Cambridge, London, 1904). Section 284 in the 3rd and subsequent editions.

8. H. Poincare, Compt. Rend. $\underline{123}, 530$ (1896).

9. P.A.M. Dirac, Proc. Roy. Soc. London, Ser. A, 133, 60 (1931).

10. For a contrary view see R.A. Carrigan, Jr., Nuovo Cimento 38, 638 (1965).

11. P.B. Price, E.K. Shirk, W.Z. Osborne and L.S. Pinsky, Phys. Rev. Lett. 35, 487 (1975).

12. L.W. Alvarez, LBL-4260 (1975); P.H. Eberhard, LBL-4289 (1975); R.L. Fleischer and R.M. Walker, Phys. Rev. Lett. 35, 1412 (1975); M.W. Friedlander, Phys. Rev. Lett. 35, 1167 (1975); and R.R. Ross, LBL-4665 (1975).

13. L.W. Alvarez, Lawrence Radiation Laboratory Physics Note 470, 1963; L.J. Tassie, Nuovo Cimento 38, 1935 (1965); and L. Vant-Hur1, Phys. Rev. 173, 1412 (1968).

14. L.W. Alvarez, P.H. Eberhard, R.R. Ross and R.D. Watt, Science 167, 701 (1970); Phys. Rev. D4, 3260 (1971); Phys. Rev. D8, 698 (1973); and P.H. Eberhard, R.R. Ross, J.D. Taylor, L.W. Alvarez and H. Oberlack, Phys. Rev. D11, 3099 (1975).

15. B. Cabrera (Ph. D Thesis, Stanford, 1975).

16. G. 't Hooft, Nucl. Phys. B79, 276 (1974) and 105, 538 (1976); and A.M. Polyakov, JETP Lett. 20, 194 (1974). 
17. For more complete reviews, see P. Goddard and D.I. 01 ive, Rep. Prog. Phys. 41, 1360 (1978); Monopoles in Quantum Field Theory, edited by N.S. Craigie, P. Goddard and W. Nahm, Twortd Scientific, Singapore, 1982); and S. Coleman, Harvard, HUTP-82/A032 (1982).

18. A.S. Goldhaber in ref. 3 .

19. T.W.B. Kibble, J. Phys. A9, 1387 (1976).

20. V. Rubakov JEPT Lett. 33, 644 (1981) and Nuc1. Phys. B203, 311 (1982); and C.G. Callan, Phys. Rev. D25, 2141 (1982) and NuCT. Phys. B203, 311 (1982).

21. F. Wilczek, Phys. Rev. Lett. 48, 1146 (1982); Y. Nambu, Chicago preprint (1983); N. Pak, C. Panagiotakopoulos and Q. Shafi, ICTP preprint (1982); B. Grossman, G. Lazarides and A. Sanda, Rockerfeller preprint (1983); and A.S. Goldhaber in ref. 3.

22. J.P. Preski11, Phys. Rev. Lett. 43, 1365 (1979).

23. E.N. Parker, Astrophys. J. 160, 383 (1970); E.N. Parker, Cosmical Magnetic Fields (Clarendon, Oxford, 1979).

24. G. Lazarides, Q. Shafi, T. Walsh, Phys. Lett. B100, 21 (1981).

25. G.S. LaRue, J.D. Phillips, and W.M. Fairbank, Phys. Rev. Lett. 46, 967 (1981) and references therein.

26. J. Schwinger, Phys. Rev. 144, 1087 (1966); 173, 1536 (1968); and Science 165, 797 (1969).

27. B. Cabrera, in ref. 3 .

28. L.P. Gorkov, Sov. Phys. JETP 9, 1364 (1959), demonstrated that the Ginzberg-Landau macroscopic description of superconductivity follows from the Bardeen-Cooper-Schriffer microscopic theory.

29. C.C. Tsuei, in ref. 3 .

30. H. Frisch, private communication.

31. M.J. Price, CERN-EP-82-8 (1982); and D. Morris, private communication.

32. A good account of these effects is contained in E. Amaldi, in 01d and New Problems in Elementary Particles, edited by G. Puppi TAcademic, New Yörk, 1978), p. I

33. S.P. Ahlen and K. Kinoshita Phys. Rev. D26, 2347 (1982) provides a very nice calculation and a guide to the iiterature. See also S.P. Ahlen in ref. 3.

34. J. Lindhard, Mat. Fys. Medd. Dan. Vid. Selsk. 28, \#8 (1954). 
35. S.D. Drel1, N.M. Krol1, M.T. Mueller, S.J. Parke and M.A. Ruderman, Phys. Rev. Lett. 50, 644 (1983).

36. B.L. Beron and R. Hofstader, Phys. Rev. Lett. 23, 184 (1969).

37. L. Sulak, T. Armstrong, H. Baranger, M. Bregman, T. Bowen, A.E. Pifer, A. Polakos, H. Bradner, A. Parvulescu, W.V. Jones and $J$. Learned, Nucl. Inst. Meth. 161, 203 (1979).

38. V.P. Martem'yanov and S.Kh. Khakimov, Sov. Phys. JETP 35, 20 (1972).

39. C.W. Akerlof, Phys. Rev. D26, 1116 (1982); and Phys. Rev. D(1983) to be published.

40. B.C. Barish, R.G. Cooper, C.E. Lane and G. Liu, CALT-68-988 1983, and Barish in ref. 3 .

41. G.B. Collins, J.R. Ficenec, D.M. Stevens, W.P. Trower and J. Fischer, Phys. Rev. D8, 982 (1973) summarizes these events.

42. M.A. Ruderman and D. Zwanziger, Phys. Rev. Lett. 22, 146 (1969).

43. D.L. Burke, H.R. Gustafson, L.W. Jones and M.L. Longo, Phys. Lett. B60, 113 (1975); G.F. Del1, H. Uto, L.C.L. Yuan, E. Amaldi, M. Beneventano, B. Borgia, P. Pistilli, I. Sestili and J. Dooher, Nuovo Cimento Lett. 15, 269 (1976); D.M. Stevens, G.B. Collins, J.R. Ficenec, W.P. Trower, J. Fischer and S. Iwata, Phys. Rev. D14, 2207 (1976); and G.F. De11, L.C.L. Yuan, L.E. Roberts, J. Dooher, E. Amaldi, M. Beneventano, B. Borgia, A. Capone, F. de Notaristefani, U. Dore, F. Ferroni, E. Longo, L. Luminari, P. Pistilli and I. Sestili,

44. C. Hil1, FERMILAB-Conf-82/71-THY, and in ref. 3 .

45. B. Aubert, P. Musset, M. Price and J.P. Vialle, CERN-EP/82-152 and P. Musset in ref. 3 .

46. K. Kinoshita, P.B. Price and D. Fryberger, Phys. Rev. Lett. 48,77 (1982).

47. E.B. Bogomol'nyi, Sov. J. Nucl. Phys. 24, 449 (1976).

48. E.N. Alexeyev, M.M. Boliev, A.E. Chudakov, B.A. Makoev, S.P. Mikheyev, and Yu.Y. Sten'kin, Nuovo Cimento Lett. 35, 413 (1982).

49. E. Loh in ref. 3 .

50. J.D. U1 Iman, Phys. Rev. Lett. 47, 289 (1981).

51. D.E. Groom, E. Loh, H.H. Nelson and D.M. Ritson, Phys. Rev. Lett. $50,573(1983)$.

52. T. Mashimo, K. Kawagoe and M. Koshiba, J Phys. Soc. Japan, 51, 3067 (1982). 
53. R. Bonarelli, P. Capiluppi, I. D'Antone, G. Giacomelli, G. Mandrioli, C. Merli and A.M. Rossi, Phys. Lett. B112, 100 (1982).

54. J. Bartelt, H. Courant, K. Heller, T. Joyce, M. Marshak, E. Peterson, K. Ruddick, M. Shupe, D.S. Avres, J.W. Dawson, T.H. Fields, E.N. May and L.E. Price, Phys. Rev. Lett. 50, 655 (1983).

55. K. Konishita and P.B. Price, Phys. Rev. D24, 1707 (1981); and P.B. Price in ref. 3 .

56. E. Goto, Prog. J. Phys. Soc. Japan. 13, 1413 (1958) and E. Goto, H.H. Kolm and K.W. Ford, Phys. Rev. 132, 387 (1963).

57. D. C1ine in ref. 3 .

58. R.A. Carrigan, Nature (London) 288, 348 (1980).

59. M.S. Turner, Chicago, EFI - 82/55 (1982).

60. M.J. Longo, Phys. Rev. D25, 2399 (1982).

61. S. Dimopoulos, S.L. Glashow, E.M. Purcell and F. Wilczek, Nature (London) 298, 824 (1982).

62. K. Freese and M.S. Turner, Chicago - EFI-82-56 (1982). See also Ref. 33.

63. J.M. Wilcox, Comments Astrophys. Space Phys. 4, 141 (1972).

64. L.W. Alvarez, private communication.

65. C. Sawyer, Solar Phys. 35, 37 (1974); M. Stix and E. Wiehr, Solar Phys. 37, 493 (1974); R. Howard, Solar Phys. 38, 283 (1974).

66. M.S. Turner, Chicago - EFI-82-52 (1982).

67. R. Davis, Jr., Proc. Brookhaven Solar Neutrino Conf., BNL 50879
(1978) p. 1.

68. J. Stone, private communication; and P.C. Bosetti, P.W. Gorhman, F.A. Harris, J.G. Learned and K. Mitsui, Dumand HDC-16-82 (1982).

69. E.W. Kolb, S.A. Colgate and J.A. Harvey, Phys. Rev. Lett. 49, 1373 (1982).

70. J.A. Harvey, Princeton, Print-82-0868 (1982); and A.S. Goldhaber, private communication.

71. D.M. Ritson, SLAC-PUB-2977 (1982).

72. E.E. Salpeter, S.L. Shapiro, and I. Wasserman, Phys. Rev. Lett. 49, $1114(1982)$ and I. Wasserman in ref. 3 . 
73. M.S. Turner, E.N. Parker and T.J. Bogdan, Phys. Rev. D26, 1296 (1982).

74. J. Arons and R.D. Blanford, Phys. Rev. Lett. 50, 544 (1983).

75. E.M. Purcell in ref. 3 .

76. P. Eberhard has proposed alternatives, private communication.

77. Y. Rephaeli and M.S. Turner, Chicago - EFI-82-37 (1982).

78. G. Lazarides and Q. Shafi, Phys. Lett. B to be published and G. Lazarides in ref. 3.

79. A.H. Guth, Phys. Rev. D23, 347 (1981); J.D. Barrow and M.S. Turner, Nature 298, 801 (1982); and A.H. Guth in ref. 3.

80. S. Coleman and E. Weinberg, Phys. Rev. D7, 1888 (1973).

81. A.D. Linde, Phys. Lett. B108, 389 (1982); A. Albrecht and P.J. Steinhardt, Phys. Rev. Lett. $48,1220(1982)$; and S.W. Hawking and I.G. Moss, Phys. Lett. B110, 35 (1982).

82. J. Ellis, Neutrino 82, CERN-TH-3354 (1982) and in ref. 3 . 


\section{FIGURE CAPTIONS}

Figure 1. Small spatial domains in the early universe coalesce to form a magnetic monopole.

Figure 2. The GUT monopole is structured in regions defined by the degree of unification and virtuar constituents. From the inside out there is a grand unification core with massive $X$ particles, the electroweak unification region with intermediate bosons $(Z, W)$, the confinement region with photons $(\gamma)$ and gluons $(g)$, and a fermion-antifermion condensate with quark-antiquark pairs.

Figure 3. Schematic of the Stanford dynamic monopole detector that found the monopole signal. This ultra-low field superconducting magnetometer is a four-turn $5-\mathrm{cm}$ diameter niobium wire ring positioned with its axis vertical. The ring, connected to a SQUID, is mounted inside an ultra-low field shield which, in turn, is mounted inside a single mumetal cylinder to provide $180 \mathrm{db}$ isolation from external magnetic field changes. (from ref. 2)

Figure 4. The monopole induced current in a superconducting ring for an axial penetrating trajectory (___ ), an off-axis penetrating trajectory (....), and for two near-miss trajectories (...) and $(\ldots) . s$ is the trajectory distance from the ring plane. (from ref.2)

Figure 5. Effect of a monopole passing through a current loop. The monopole effectively draws flux through the loop and leaves a 
residual toroidal field around it. The inset shows how the flux lines around the loop pinch off to form closed circles. (from ref. 28)

Figure 6 . Predicted energy loss rate to silicon for protons and magnetic monopoles as a function of $B$. The lines represent predictions while the circles are averages of proton measurements. The hydrogen calculation includes the effect of Zeeman splitting. A threshold, $T$, as shown for silicon, can cut off the energy loss. Reference escape velocities from the earth, sun, and galaxy are shown. (refs. 33-35)

Figure 7. Upper limits (95\% CL) for accelerator and colliding beam produced monopoles versus monopole mass. Only the lowest limits up to a particular monopole mass are shown. (refs. 2, 45 and 46 )

Figure 8. Limits for cosmic-ray flux of GUT monopoles ( $95 \% \mathrm{CL}$ ) plotted versus the $\beta$ monopole. The Stanford result corresponds to one candidate event. All other detectors relied on ionization. Also shown for monopoles whose mass is $10^{16} \mathrm{GeV} / \mathrm{c}^{2}$ are the Parker galatic bound $(\cdots)$ and upper limits based on the expansion of the universe for monopoles distributed uniformly and clumped in galaxies (-..-). (refs, 48-55) 


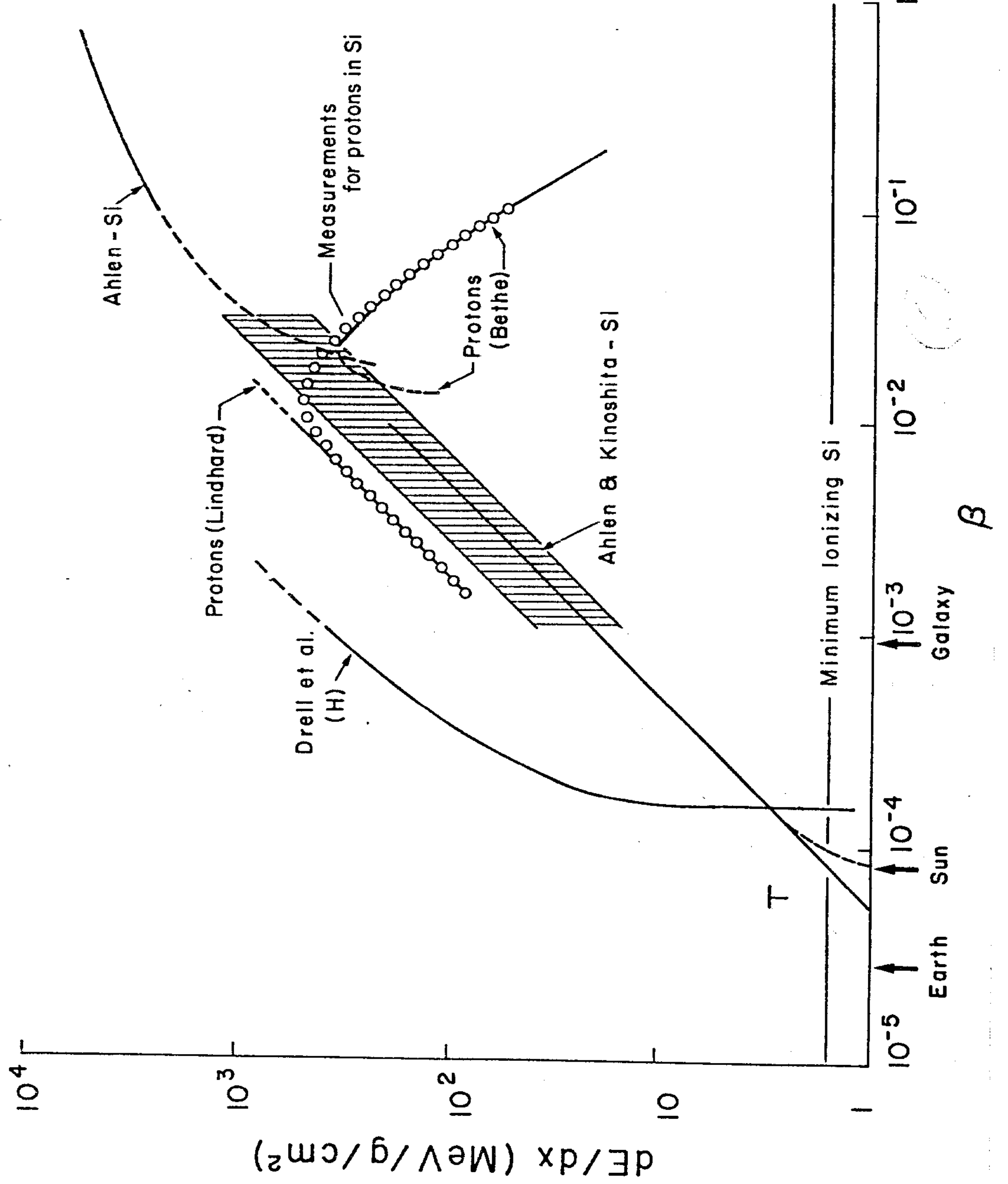




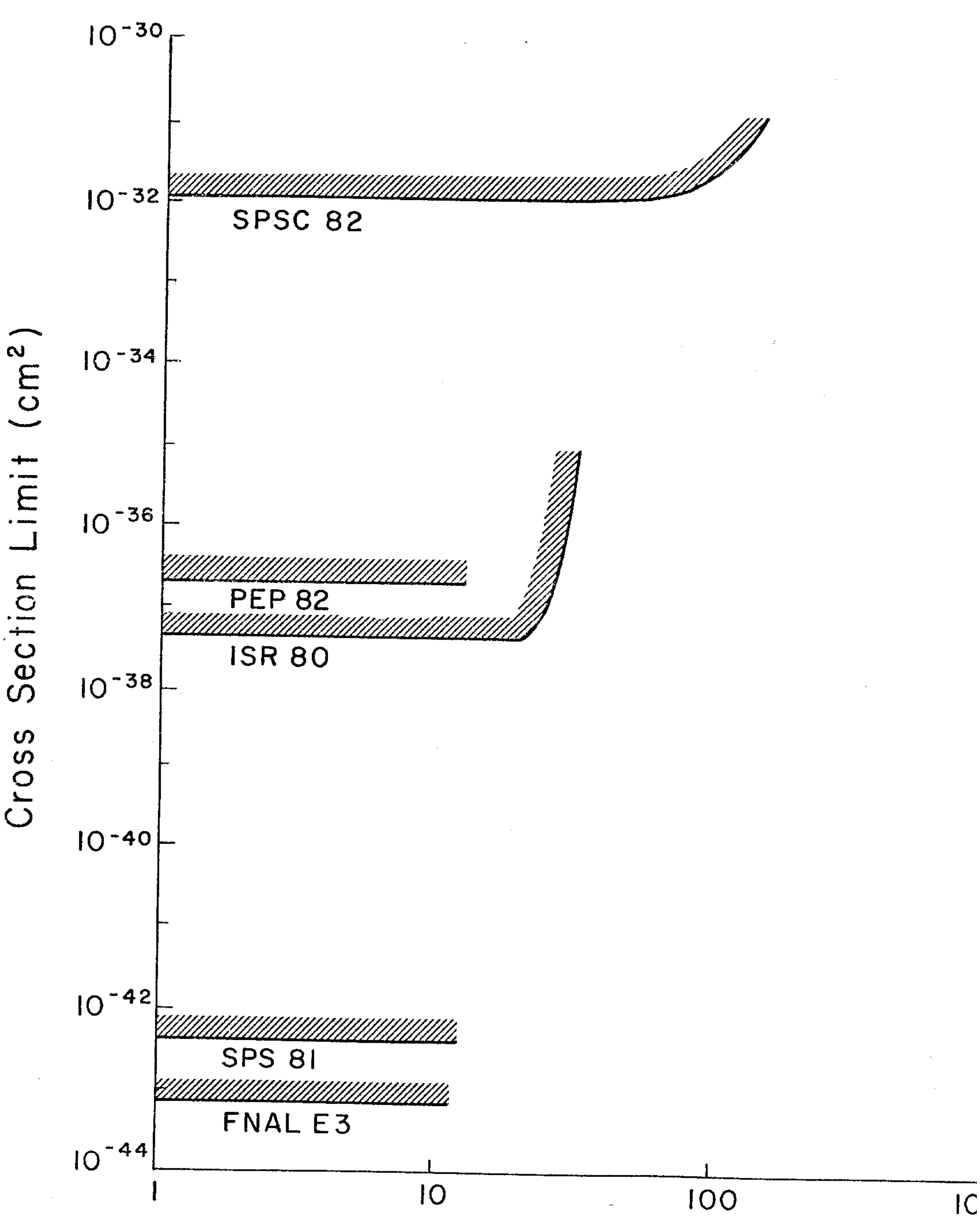

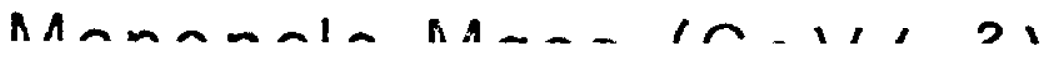

